\title{
Image Correction and Reconstruction for Breast Biopsy
}

\begin{abstract}
A methodology for enhancing image quality for a given biopsy sensor is presented. Due to the specific characteristics of the sensor the raw image quality is non optimal. We propose an approach for image enhancement based on flat field intensity normalisation and detection and correction of dead pixels effects. Qualitative and quantitative evaluation is provided using phantom images in order to show the validity of the method.
\end{abstract}

\section{Background}

The work presented here is part of an ongoing research project which aims to develop a stereotaxic breast biopsy system using real time digital acquisition based on the newly developed detector. The system will operate with significantly lower doses of radiation compared to current mammographic systems and, moreover, acquired images will show better spatial resolution and higher dynamic range compared with the added advantage of a real-time acquisition.

The current prototype uses a commercially available dynamic imaging x-ray camera (DIC100T, AJAT OY Ltd) which has similar characteristics to the detector under development. The DIC100T camera incorporates $8 \mathrm{CdTe}$ detectors, with a total area of $508 * 512$ pixels with $100 \mu \mathrm{m}$ of pixel size. The acquired images present two main problems that affect image quality: the different response of the detectors and the presence of abnormal pixels. Different enhancement proposals can be found in the literature related to X-ray imaging [2,4] and more specifically to mammography [5]. A common approach is to apply algorithms based on flat field correction filters and specific image restoration techniques depending on the characteristics of the sensor $[1,4,5]$.

This paper presents a methodology for enhancing image quality for a mammographic detector focusing on intensity normalisation and minimisation of dead pixel effects. Qualitative and quantitative evaluation is provided in order to show the validity of the method.

\section{Methodology}

The proposed method is based on a initial normalization of the image. Subsequently dead pixels are detected (pixels with significantly different intensity values compared to its neighbors). Finally, linear intensity interpolation is applied to those pixels using information from the neighbouring pixels. Figure 3(a) shows a raw image directly from the detector, where dead pixels are clearly shown in rows and columns but also randomly distributed. In addition, sensors show different responses, illustrating the need for normalisation. 


\subsection{Flat Field Correction}

As described earlier, a different response is obtained for the eight sensors. In order to normalize the response of the different pixels we will use a flat-field correction approach [4]. The method to correct the raw acquired data is defined by the following equation,

$$
I_{C}=I_{A v} \frac{\left(I_{O}-\overline{I_{N X R}}\right)}{\overline{I_{X R}}-\overline{I_{N X R}}}
$$

where $I_{C}$ is the corrected image, $I_{O}$ is the original image to be corrected, $\overline{I_{N X R}}$ is the average of a set of dark field (offset) images (with non x-ray exposure), $\overline{I_{X R}}$ is the average of a set of flat-field (gain) images (with x-ray exposure but no object present) and $I_{A v}$ is the average of the difference $\overline{I_{X R}}-\overline{I_{N X R}}$ or $\overline{I_{X R}}$.

\subsection{Dead Pixel Detection and Correction}

A common problem in X-ray detection is the presence of pixels which have an abnormal response, mainly due to defects or imperfections in the sensor (randomly distributed) or to the inherent physical constraints of the detector (spacing between the sensors). Those pixels are often referred to as dead pixels. In order to detect those pixels, the mean $(\mu)$ and the standard deviation $(\sigma)$ is computed from the flat field corrected images (images with x-ray exposure and no object). We will define as dead pixels those pixels with a value larger than $\mu \pm k$.

Once dead pixels have been detected, two different approaches are used to correct their intensity, differentiating between randomly distributed dead pixels (RD) and dead pixels due to detector spacing (DS)(see Fig. 1(a)). RD pixels are corrected by using a standard 3-by-3 average filter taking into account non-dead pixels values [3], whereas DS pixels are corrected using a specific linear interpolation approach. Due to sensor layout, acquired images show obvious rows and columns of dead pixels. In that case, averaging or other generic approaches obtained unsatisfactory results as not only dead pixels but also neighbor pixels are affected showing a slightly lower intensity profile (blooming effect). An interpolation approach, inspired by the work in [2] is used here and is illustrated in Fig. 1(b). Dead and their neighbor pixels (marked in red in the figure) are corrected using $2 \mathrm{D}$ interpolation values from a $2 \mathrm{D}$ local neighborhood (blue pixels).

\section{Results}

Our approach has been evaluated using a total set of 100 images. From this set, 20 are dark-field images $\left(I_{N X R}\right), 20$ flat-field images $\left(I_{X R}\right), 20$ images of a grid, 20 images of a breast phantom and 20 images of a integrated circuit.

Flat-field Correction We have evaluated the effect of varying the different parameters in the flat-field equation (Eq. 2.1) using the 20 dark-field and flatfield images, and different average methods (median and mean). Figure 2(a) 


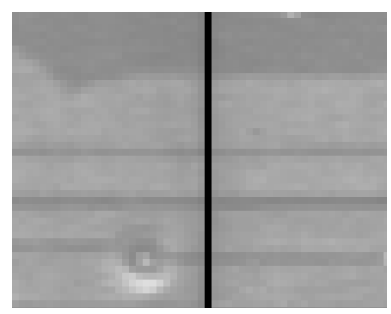

(a)

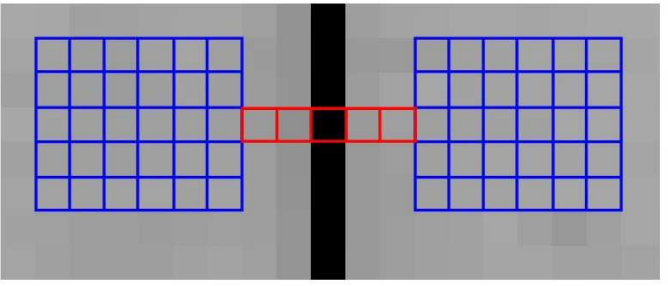

(b)

Fig. 1. Dead pixel correction: (a) Dead pixels due to detector spacing and (b) a blown up region illustrating the interpolation approach.

shows those results where $\overline{I_{N X R}}=0$ in $a, b, c, d, \overline{I_{N X R}}=$ mean in $e, f, g, h$, $\overline{I_{N X R}}=$ median in $i, j, k, l, \overline{I_{X R}}=$ median in $c, d, i, j, k, l, \overline{I_{X R}}=$ mean in $a, b, e, f, g, h, I_{A v}=$ median $\left(\overline{I_{X R}}-\overline{I_{N X R}}\right)$ in $a, c, g, k, I_{A v}=\operatorname{mean}\left(\overline{I_{X R}}-\overline{I_{N X R}}\right)$ in $b, d, h, l, I_{A v}=\operatorname{median}\left(\overline{I_{X R}}\right)$ in $e, i, I_{A v}=\operatorname{mean}\left(\overline{I_{X R}}\right)$ in $f, j$. From the results, there is no significant difference between using mean or the median in the different parameters, only if we use the $I_{A v}$ as the average of $\overline{I_{X R}}$ we obtain high deviations.

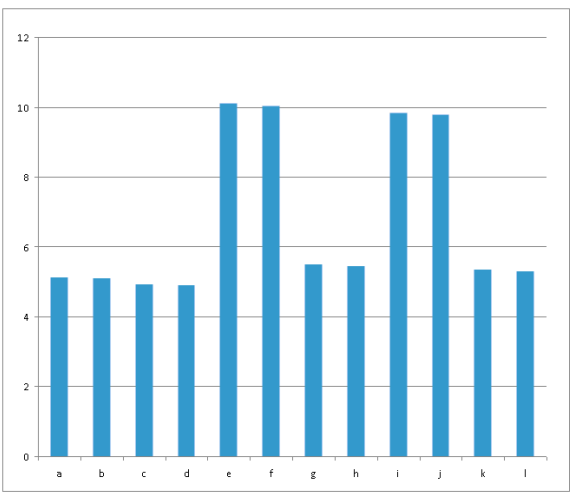

(a)

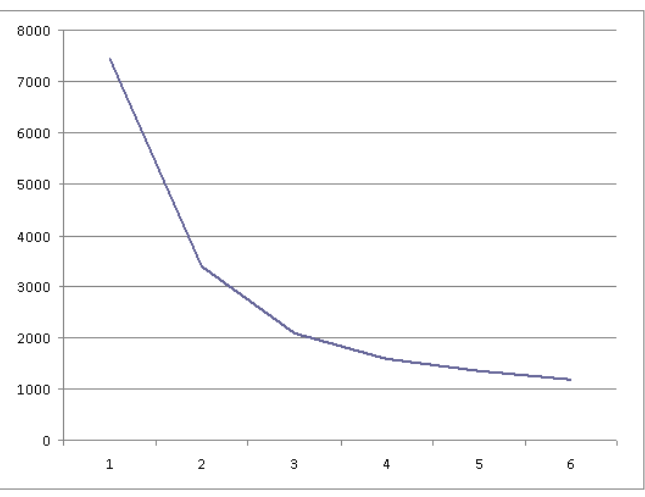

(b)

Fig. 2. Evaluation results: (a) Mean standard deviation when varying parameters in the flat-field correction equation (see main text) and (b) dead pixel detection as a function of the $k$ value.

Dead pixel detection We have tested different values of $\mathrm{k}$ in order to identify dead pixels. If the $\mathrm{k}$ value is underestimated, some normal pixels will be treated as dead pixels, in the other hand, if the value is high, we can miss dead pixels. Common statistical outlier definitions of $k \geq 3$ provide a good trade-off for detection rates as shown in Fig 2(b). 
Finally, Fig. 3 shows (a) the original detector image and (b) the results of applying the proposed enhancement. Note that detector intensity has been corrected and dead pixels successfully recovered.

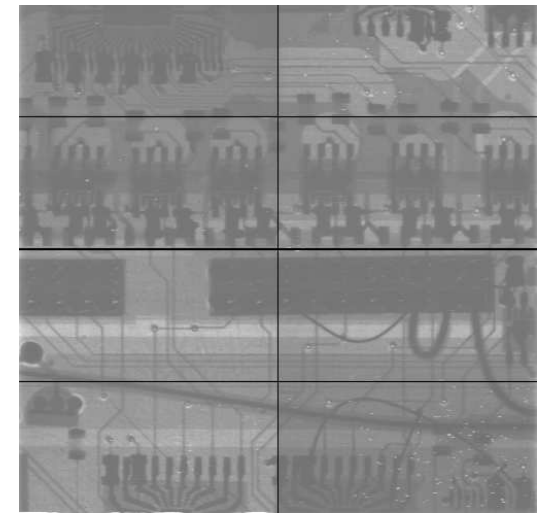

(a)

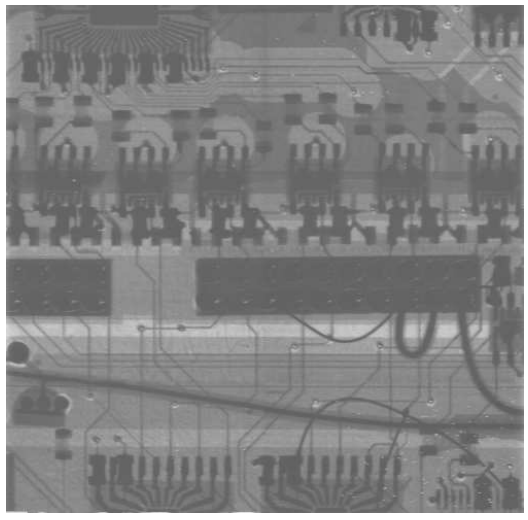

(b)

Fig. 3. Enhancement results: (a) original and (b) corrected images.

\section{Discussion and Conclusions}

An image enhancement methodology for biopsy stereotactic images has been presented based on applying flat field intensity correction techniques and a specific algorithm for dead pixel detection and correction. Quantitative evaluation of the different aspects of the method have been provided. In addition, enhancement results have been presented which shows the effectiveness of our approach. Future work will focus on larger scale evaluation of the methodology, including more clinical quality assurance aspects (i.e. using radiological phantoms). In addition, and once the final detector is available, the enhancement method will be applied and evaluated with new data.

\section{References}

1. T. Han, D. G. Goodenough, A. Dyk, and J. Love: Detection and Correction of Abnormal Pixels in Hyperion Images. IGARSS 2002, vol. III, pp. 1327-1330, 2002.

2. J. Jakubek: Data processing and image reconstruction methods for pixel detectors. Nuclear Instruments and Methods A 576 (2007) 223-234.

3. A. Kokaram R.D., Morris, W.J. Fitzgerald P.J.W. Rayner: Interpolation of Missing Data in Image Sequences. IEEE Trans. Image Processing 4(11) (1995) 1509-1519.

4. ALC Kwan, JA Seiber, JM Boone JM: An improved method for flat-field correction of flat panel x-ray detectors. Med Phys 33 (2006) 391-393.

5. M.B. Williams, P.A. Mangiafico and P.U. Simoni: Noise power spectra of images from digital mammography detectors. Med. Phys. 26 (1999) 1279-1293. 